\title{
CHARACTERIZATIONS OF GENERALIZED UNISERIAL ALGEBRAS. II
}

DRURY W. WALL

Let $\mathfrak{A}$ be a finite dimensional algebra with unit element over a field. $\mathfrak{A}$ is generalized uniserial if every primitive (left or right) ideal has a unique composition series. $\mathfrak{A}$ is a UMFR algebra (an algebra with a unique minimal faithful representation) if $\mathfrak{A}$ has only one faithful representation which is minimal with respect to being faithful. The notation used in this paper will be that of an earlier paper [5] in which subclasses of the UMFR algebras were studied. In this notation, $\mathfrak{A}$ is of type $\mathrm{ABC}$ if every primitive ideal is subordinate to a dominant ideal, and type $B$ if every primitive ideal is weakly subordinate to a dominant ideal. It is known [3, Theorem 5] that an algebra is UMFR if and only if every primitive ideal is weakly subordinate to a set of dominant ideals. In other papers $[3 ; 4]$, the names QF-2, QF-3* and QF-3 have been used for ABC, B and UMFR, respectively. For further details concerning these classes see the paper by R. M. Thrall [3] and the author's previous papers $[4 ; 5]$. For the definitions of other terms see, in addition to these papers, either of the references on ring theory $[1 ; 2]$.

The purpose of this paper is to extend an earlier result, namely: An algebra $\mathfrak{A}$ is generalized uniserial if and only if, for every twosided ideal $\mathfrak{Z}$ of $\mathfrak{A}$, the residue class algebra $\mathfrak{A} / \mathfrak{Z}$ is type $\mathrm{B}[4$, Theorem 3 ]. It will be shown here that an algebra is generalized uniserial if and only if all of its residue class algebras are UMFR.

THEOREM 1. If $\mathfrak{A}$ is a UMFR algebra which is not type $B$ then there exists a two-sided ideal $\mathfrak{3}$ of $\mathfrak{A}$ such that $\mathfrak{A} / \mathfrak{B}$ is not UMFR.

Proof. Let $\mathfrak{A}$ be UMFR but not type B. Thus, there is either a primitive left ideal or a primitive right ideal which is weakly subordinate to a set of dominant ideals but is not weakly subordinate to any single dominant ideal. Consider here the left ideal case. (The proof for the right ideal case would be similar.) Thus, there is a left ideal $\mathfrak{R}$ which is weakly subordinate to a set $\left\{\mathfrak{D}_{i}\right\}_{i=1}^{r}$ of mutually nonisomorphic dominant ideals of $\mathfrak{A}[5$, Theorem 2]. This means that there exists a set of ideals $\left\{\mathfrak{D}_{i j}\right\}_{i=1, j=1}^{r(i)}$ such that, for each $i$ and $j$, $\mathfrak{D}_{i j}$ is a subideal of $\mathfrak{D}_{i}$ and $\mathfrak{R}$ is isomorphic as an $\mathfrak{A}$-module to a submodule of the $\mathfrak{A}$-module $\sum_{i=1}^{r} \sum_{j=1}^{o(t)} \mathfrak{D}_{i j}$, the direct sum of the $\mathfrak{D}_{i j}$. Note that since $\mathfrak{A}$ is not type $\mathrm{B}, r>1$. However, the proof given here

Presented to the Society January 28, 1958; received by the editors April 28, 1958. 
holds if $\mathfrak{A}$ is only assumed to be not of type ABC. It is essential that for some $i, g(i)>1$.

Let $\beta$ be the set of all primitive ideals $\subseteq$ of $\mathfrak{A}$ which are either weakly subordinate to the dominant ideal $\mathfrak{D}_{1}$ or weakly subordinate to some set of mutually nonisomorphic dominant ideals of which one is $\mathfrak{I}_{1}$. Note that $\mathfrak{R}$ is an element of $\boldsymbol{\rho}$. Partially order $\boldsymbol{P}$ by defining, for $\mathfrak{S}_{1}=\mathfrak{A} e_{1}$ and $\mathfrak{S}_{2}=\mathfrak{A} e_{2}$ in $\mathcal{P}, \mathfrak{S}_{1}<\mathfrak{S}_{2}$ if and only if in every composition series of $\mathfrak{D}_{1}$ there is a constituent isomorphic to $\mathfrak{A} e_{2} / \mathfrak{N} e_{2}$ which is "above" every constituent isomorphic to $\mathfrak{A} e_{1} / \mathfrak{N} e_{1}$. There must exist some element in $\mathcal{P}$ which is maximal with respect to this partial ordering. Let $\mathfrak{S}_{0}$ be such a maximal element.

It is possible that $\Xi_{0}$ is weakly subordinate to the dominant ideal $\mathfrak{D}_{1}$. In this case, the method of proof used in the earlier result [4, Theorem 3] and the construction therein show that there is a twosided ideal $\mathbb{Z}$ such that $\mathfrak{X} / \mathbb{Z}$ is not UMFR. However, the proof below is general enough to include this case.

If $\mathfrak{S}_{0}$ is not weakly subordinate to a single dominant ideal, then without loss of generality it can be assumed that the original $\&$ was the maximal element $\mathfrak{S}_{0}$. Thus, we assume that $\mathfrak{R}=\mathfrak{A} e$ is such that every constituent which always occurs above all constituents isomorphic to $\mathfrak{A} e / \mathfrak{N} e$ in composition series of $\mathfrak{D}_{1}$ is isomorphic to the top constituents of dominant or subordinate ideals.

Let $\mathcal{G}=\{\mathfrak{M} \mid \mathfrak{M}$ is a primitive ideal of $\mathfrak{A}$ such that: (1) $\mathfrak{M}$ is subordinate to or isomorphic to $\mathfrak{D}_{1}$ and, (2) $\mathbb{R}$ is weakly subordinate to $\left.\left\{\mathfrak{M}, \mathfrak{D}_{2}, \cdots, \mathfrak{D}_{r}\right\}\right\}$. Choose a subset $\left\{\mathfrak{R}_{i}\right\}_{i=1}^{n}$ of $\mathcal{G}$, maximal with respect to having the generating idempotents mutually orthogonal. Thus, the $\mathfrak{R}_{i}$ can be considered as components of a decomposition of $\mathfrak{U}$ into a sum of primitive left ideals.

For each $\Omega_{i}$, let $\Omega_{i}^{\prime}$ be its unique minimal subideal. For each $i, \Omega_{i}^{\prime} \mathfrak{A}$ is a two-sided ideal of $\mathfrak{A}$ which is the union of all minimal subideals of ideals which are isomorphic to $\mathfrak{R}_{i}[4$, Lemma 4$]$. Since $\mathfrak{R}$ was chosen as a maximal element of $\boldsymbol{P}$, it is impossible for any of the $\mathfrak{R}_{i}^{\prime} \mathfrak{A}$ to contain elements of a nonsubordinate primitive ideal. If $\mathfrak{A} f$ were such an ideal then it would have a subideal isomorphic to $\mathfrak{R}_{i}$. This would imply that in some composition series of $\mathfrak{D}_{1}$ a constituent isomorphic to $\mathfrak{U} f / \mathfrak{R} f$ would be above all constituents isomorphic to $\mathfrak{A} e_{\boldsymbol{i}} / \mathfrak{N} e_{\boldsymbol{i}}$, where $\mathfrak{R}_{i}=\mathfrak{A} e_{i}$. But, since $\mathfrak{R}$ is weakly subordinate to $\left\{\mathfrak{R}_{i}, \mathfrak{D}_{2}, \cdots, \mathfrak{D}_{r}\right\}$, a constituent isomorphic to $\mathfrak{A} e_{i} / \mathfrak{M} e_{i}$ is always above all constituents isomorphic to $\mathfrak{A} e / \mathfrak{N} e$. This would contradict the maximality of $\mathfrak{R}$.

Let $\mathbb{Z}$ be the sum of the $\mathfrak{R}_{i}^{\prime} \mathfrak{A}$. Since the set $\left\{\mathfrak{R}_{i}\right\}_{i=1}^{n}$ was chosen maximal and since every primitive ideal of $\mathfrak{A}$ is at least weakly sub- 
ordinate, $\mathbb{Z}$ can be written as the direct sum of the $R_{i}^{\prime}$. Moreover, by the construction of $\mathbb{Z}, \mathfrak{R} \cap \mathbb{Z}=0$. Thus, $R / R \cap Z \cong R$.

Assume that the residue class algebra $\mathfrak{A} / \mathfrak{Z}$, denoted by $\mathfrak{A}^{*}$, is a UMFR algebra. If $\mathfrak{R}^{*}$ is the ideal $\mathfrak{\Omega} / \mathfrak{\Omega} \cap \mathbb{Z}$ of $\mathfrak{A}^{*}$ then $\mathfrak{\Omega}^{*}$ is either a dominant ideal of $\mathfrak{A}^{*}$, is subordinate to a dominant ideal of $\mathfrak{A}^{*}$, or is weakly subordinate to a dominant ideal of $\mathfrak{A}^{*}$ or to a set of dominant ideals of $\mathfrak{A}^{*}$. But, since $\mathfrak{\imath}^{*} \cong \mathfrak{R}, \mathfrak{R}^{*}$ has more than one mininal subideal and cannot be either dominant or subordinate in $\mathfrak{A}^{*}$. Thus $\Omega^{*}$ must be weakly subordinate to some set $\left\{\mathfrak{D}_{1}^{*}\right\}_{i=1}^{s}, s \geqq 1$, of mutually nonisomorphic dominant ideals of $\mathfrak{A}$.

The socle $\mathfrak{S}(\mathbb{R})$ of $\mathfrak{R}$, the union of all minimal subideals of $\mathfrak{R}$, can be written $\sum_{i=1}^{r} \sum_{j=1}^{o(i)} \mathfrak{M}_{i j}$, where for each $i$, each $\mathfrak{M}_{i j}$ is isomorphic to the unique minimal subideal of $\mathfrak{D}_{i}$. Similarly, the socle $\mathfrak{S}\left(\Omega^{*}\right)$ of $\mathfrak{R}^{*}$ can be written $\sum_{i=1}^{s} \sum_{j=1}^{h(1)} \mathfrak{M}_{i j}^{*}$, where for each $i$, each $\mathfrak{M}_{i j}^{*}$ is isomorphic to the unique minimal subideal of $\mathfrak{D}_{i}^{*}$. However, since $\mathfrak{Z} \cong \mathbb{R}^{*}, r=s$ and the $\mathfrak{D}_{i}^{*}$ can be arranged so that for each $i, g(i)=h(i)$. Thus, there must be one of the $\mathfrak{D}_{i}^{*}$, denote it by $\mathfrak{D}^{*}$, whose unique minimal subideal $\mathfrak{M}^{*}$ is isomorphic to $\mathfrak{M}_{1 j}, j=1, \cdots, g(1)$ and, hence, isomorphic to the unique minimal subideal of $\mathfrak{D}_{1}$.

If $f^{*}$ is the generating idempotent of $\mathfrak{D}^{*}$, i.e., $\mathfrak{A}^{*} f^{*}=\mathfrak{D}^{*}$, let $f$ be a primitive idempotent of $\mathfrak{A}$ such that $f \in f^{*}$. If $\mathfrak{D}=\mathfrak{A} f$ then $\mathfrak{D}$ is a primitive ideal of $\mathfrak{A}$ such that $\mathfrak{D} / \mathfrak{D} \cap \mathbb{Z}=\mathfrak{D}^{*}$. Since $\mathfrak{A}$ is a UMFR algebra, $\mathfrak{D}$ must be dominant, subordinate or weakly subordinate in $\mathfrak{A}$. Consider now the various possible cases.

CAse 1 . Assume $\mathfrak{D}$ is a dominant ideal of $\mathfrak{A}$ and let $\mathfrak{M}$ be the unique minimal subideal of $\mathfrak{D}$.

Subcase a. Assume $\mathfrak{D} \not \mathcal{D} \mathfrak{D}_{1}$. In this case, from the construction of $\mathfrak{B}, \mathfrak{D} \cap \mathbb{B}=0$. This implies that $\mathfrak{D}^{*} \cong \mathfrak{D}$ and, hence, $\mathfrak{M}^{*} \cong \mathfrak{M}$. Since $\mathfrak{M}^{*} \cong \mathfrak{M}_{i j}$, this implies $\mathfrak{M} \cong \mathfrak{M}_{i j}$, which, in turn, implies that $\mathfrak{D} \cong \mathfrak{D}_{1}$. Thus, Case 1, subcase $a$, is impossible.

Subcase b. Assume $\mathfrak{D} \cong \mathfrak{D}_{1}$. In this case, form the construction of $\mathfrak{B}, \mathfrak{D} \cap \mathfrak{B}=\mathfrak{M}$. Thus, $\mathfrak{D}^{*}=\mathfrak{D} / \mathfrak{M}$ and both $\mathfrak{D}$ and $\mathfrak{D}^{*}$ have unique minimal subideals and these subideals are isomorphic. It is known [4, Lemma 8] that if a dominant ideal has, in addition to a unique minimal subideal, a unique second constituent and these constituents are isomorphic then it has only one composition series and all of its constituents are isomorphic. Thus, all constituents of $\mathfrak{D}$ are isomorphic and, hence, no primitive ideal which is not isomorphic to $\mathfrak{D}$ can be subordinate to $\mathfrak{D}$ or weakly subordinate to any set of dominant ideals containing $\mathfrak{D}$. For if $\mathfrak{A} e^{\prime}$ were such a primitive ideal, then $\mathfrak{D}$ would have a constituent isomorphic to $\mathfrak{A} e^{\prime} / \mathfrak{N} e^{\prime}$. This contradicts 
the assumption that $\mathbb{R}$ is weakly subordinate to $\left\{\mathfrak{D}_{i}\right\}_{i=1}^{r}$. Thus, Case 1 , subcase $b$, is impossible.

CAse 2. Assume $\mathfrak{D}$ is subordinate to a dominant ideal $\mathfrak{B}$ of $\mathfrak{A}$. By arguments similar to those used in Case 1 it can be shown that it is impossible that (a) $\mathfrak{B} \not \mathfrak{D}_{1}$ or (b) $\mathfrak{B} \cong \mathfrak{D}_{1}$. Thus, Case 2 is impossible.

CASE 3 . Assume $\mathfrak{D}$ is weakly subordinate to a single dominant ideal $\mathfrak{B}$ to $\mathfrak{A}$. The possibility that $\mathfrak{B} \cong \mathfrak{D}_{1}$ can be ruled out since this would imply $\mathfrak{D} \cap \mathfrak{B}=0$ and, hence, $\mathfrak{D}^{*}$ would have more than one minimal subideal. Thus, $\mathfrak{B}$ can be chosen to be $\mathfrak{D}_{1}$. But since $\mathfrak{Q}^{*}$ is weakly subordinate to a set of dominant ideals containing $\mathfrak{D}^{*}$, there is always a constituent of $\mathfrak{D}^{*}$, namely the top constituent $\mathfrak{A} f^{*} / \mathfrak{R} f^{*}$ which is above all constituents isomorphic to $\mathfrak{A} e^{*} / \mathfrak{N} e^{*}$. But since $\mathfrak{A} f^{*} / \mathfrak{R} f^{*}$ $\cong \mathfrak{A} f / \mathfrak{N} f$ and $\mathfrak{H} e^{*} / \mathfrak{N} e^{*} \cong \mathfrak{H} e / \mathfrak{M} e$, there will be in any composition series of $\mathfrak{D}_{1}$ a constituent isomorphic to $\mathfrak{A} f / \mathfrak{N} f$ above any constituent isomorphic to $\mathfrak{P} e / \mathfrak{R} e$. This would contradict the maximality of $\mathbb{R}$ and, hence, Case 3 is impossible.

CASE 4 . Assume $\mathfrak{D}$ is weakly subordinate to a set of dominant ideals of $\mathfrak{A}$. Thus, $\mathfrak{D}$ has more than one minimal subideal and, in addition, not all of these minimal subideals are isomorphic. But $\mathfrak{D}^{*}=\mathfrak{D} / \mathfrak{D} \cap \mathbb{Z}$ has a unique minimal subideal $\mathfrak{M}^{*}$ and $\mathfrak{M}^{*}$ is isomorphic to the unique minimal subideal of $\mathfrak{D}_{1}$. Also, $\mathfrak{Z}$ is a union of minimal subideals isomorphic to the unique minimal subideal of $\mathfrak{D}_{1}$. Thus, any minimal subideal of $\mathfrak{D}$ which is not isomorphic to $\mathfrak{M}^{*}$ cannot be contained in or intersect with 3 . But, this would make such a minimal subideal of $\mathfrak{D}$ also a minimal subideal of $\mathfrak{D}^{*}$, which is impossible. Hence, Case 4 is impossible.

Thus, the primitive ideal $\mathfrak{D}$ is neither dominant, subordinate to a dominant ideal, nor weakly subordinate to a dominant ideal or to a set of dominant ideals of $\mathfrak{A}$. This would imply that $\mathfrak{A}$ is not a UMFR algebra, which contradicts the hypothesis of the theorem. Therefore, $\mathfrak{A} / 3$ is not a UMFR algebra.

Theorem 2. An algebra $\mathfrak{A}$ is generalized uniserial if and only if for every two-sided ideal 3 , the residue class algebra $\mathfrak{A} / 3$ is UMFR.

Proof. By an earlier result [4, Theorem 3] it is known that an algebra $\mathfrak{A}$ is generalized uniserial if and only if, for every two-sided ideal $Z, \mathfrak{U} / \mathbb{Z}$ is type $\mathrm{B}$. From this the "only if" part of Theorem 2 follows immediately by noting that the algebras of types $B$ are of a subclass of the UMFR algebras. Theorem 1 shows that if an algebra $\mathfrak{A}$ is such that every $\mathfrak{A} / \mathfrak{Z}$ is UMFR then $\mathfrak{A}$ is type $B$. Let $\mathscr{Z}$ be any two-sided ideal of $\mathfrak{A}$ and let $\mathfrak{A}^{*}=\mathfrak{A} / \mathfrak{Z}$. Let $\mathfrak{Z}^{*}$ be any two-sided ideal of $\mathfrak{A}^{*}$. Then, if $\mathfrak{Z}^{\prime}=\left\{x \in \mathfrak{N} \mid x+\mathfrak{Z} \in \mathfrak{Z}^{*}\right\}$ then $\mathfrak{Z}^{\prime}$ is a two-sided ideal 
of $\mathfrak{A}$ and $\mathfrak{A} / \mathfrak{Z}^{*} \cong \mathfrak{A} / \mathfrak{Z}^{\prime}$. Thus, if for all two-sided ideals $\mathbb{Z}$ of $\mathfrak{A}, \mathfrak{A} / \mathbb{Z}$ is UMFR, then for all two-sided ideals $\mathfrak{Z}^{*}$ of $\mathfrak{A}^{*}, \mathfrak{A}^{*} / \mathfrak{Z}^{*}$ is UMFR. Hence, applying Theorem 1 to $\mathfrak{A} / \mathscr{Z}, \mathfrak{A} / \mathscr{Z}$ is type $\mathrm{B}$. Therefore, all $\mathfrak{A} / \mathbb{B}$ are of type $\mathrm{B}$ and by the earlier result this implies that $\mathfrak{A}$ is generalized uniserial.

This theorem, together with the fact that every residue class algebra of a generalized uniserial algebra is generalized uniserial, establishes the following: If $P$ and $Q$ are subclasses of the class of UMFR algebras which contain the generalized uniserial algebras as a subclass (see [5]) then for an algebra $\mathfrak{A}$ the following are equivalent: (1) Every $\mathfrak{A} / \mathscr{Z}$ is in class $P$; (2) Every $\mathfrak{A} / \mathscr{Z}$ is in class $Q ;(3) \mathfrak{U}$ is generalized uniserial.

\section{BIBLIOGRAPHY}

1. E. Artin, C. J. Nesbitt, and R. M. Thrall, Rings with minimum condition, University of Michigan Publications in Mathematics, no. 1, 1944.

2. N. Jacobson, Structure of rings, Amer. Math. Soc. Colloquium Publications, vol. $37,1956$.

3. R. M. Thrall, Some generalizations of quasi-Frobenius algebras, Trans. Amer. Math. Soc. vol. 64 (1948) pp. 173-183.

4. D. W. Wall, Characterizations of generalized uniserial algebras, Trans. Amer. Math. Soc., to appear.

5. - Algebras with unique minimal faithful representations, Duke Math. J. vol. 25 (1958) pp. 321-329.

University of North Carolina 